\title{
Opening Symposium
}




\section{Air pollution and thrombosis}

\section{Air pollution, blood clotting and cardiovascular disease: evidence from epidemiological studies and impact on the population}

\section{Annette Peters}

Helmholtz Zentrum München - German Research Center for Environmental Health, Institute of Environmental Epidemiology, Neuherberg, Germany; Harvard School of Public Health, Department of Environmental Health, Boston, MA, USA.

Ambient particulate matter has been associated with an increased risk for hospital admission and mortality due to cardiovascular disease. This could be observed in association with short-term fluctuations of ambient fine particles (particulate matter smaller than $2.5 \mu \mathrm{m}$ (PM2.5)) and with elevated annual average concentrations of PM2.5. Ischemic heart disease has been particularly shown to increase with both short-term and long-term exposure to ambient particles. The American Heart Association has summarized the evidence from population, clinical and experimental studies in a scientific statement (1). Systemic inflammatory responses as well as translocation of ultrafine particles (particles with a diameter smaller than $100 \mathrm{~nm}$ ) are being discussed as the possible mechanisms responsible for triggering acute myocardial infarction. The association between ambient particulate matter and the onset of myocardial infarction and the role of elevated ambient concentrations of fine and ultrafine particle in inducing pro-thrombotic states will be highlighted.

\section{Reference}

1. Brook RD, Rajagopalan S, Pope CA, III, Brook JR, Bhatnagar A, Diez-Roux AV, Holguin F, Hong Y, Luepker RV, Mittleman MA, Peters A, Siscovick D, Smith SC, Jr., Whitsel L, Kaufman JD. Particulate Matter Air Pollution and Cardiovascular Disease. An Update to the Scientific Statement From the American Heart Association. Circulation 2010; May 10 [Epub ahead of print] 


\title{
Beyond the DNA sequence: epigenetic mechanisms of prothrombotic air pollution effects
}

\author{
Andrea Baccarelli
}

Università degli Studi di Milano - Fondazione IRCCS Ca' Granda Ospedale Maggiore Policlinico, Milan, Italy

Epidemiological data have consistently linked exposure to air pollution, particularly from vehicular traffic, with morbidity and mortality from heart disease. Recent investigations have shown that air pollution exposure may also increase the risk of venous thromboembolism. Traffic-related air pollution consists of both gaseous and particulate-matter pollutants. The former include nitrogen dioxide, benzene, and sulfur dioxide. The latter consist of particulate matter (PM) of varying aerodynamic diameters. Because of their small size, these particles can be inhaled deeply into the lungs and deposited in the alveoli. Recent evidence has suggested that epigenetic mechanisms might be in the path linking air pollution inhalation with cardiovascular disease. Epigenetics investigates heritable changes in gene expression that occur without changes in DNA sequence. Several epigenetic mechanisms, including DNA methylation and histone modifications, can change genome function under exogenous influence. Methylation of $\mathrm{CpG}$ dinucleotides, the best understood of the epigenetic mechanisms, is generally associated with gene silencing and is maintained through cellular division. The presentation will review current evidence indicating that epigenetic alterations mediate effects caused by exposure to air pollution.

Current evidence indicates that air pollutants, including benzene and PM, can alter epigenetic marks, and that the same or similar epigenetic alterations can be found in patients with the disease of concern. Available evidence suggests that epigenetics holds substantial potential for furthering our understanding of the molecular mechanisms activated by of environmental risk factors, as well as for predicting health-related risks due to conditions of environmental exposure and individual susceptibility. 


\title{
Inhaled particles and thrombosis: a clinical toxicology perspective
}

\author{
Nicholas L. Mills ${ }^{1}$, Andrew J. Lucking ${ }^{1}$, Nicholas A. Boon ${ }^{1}$, Thomas Sandstrom ${ }^{2}$, \\ Anders Blomberg ${ }^{2}$, Ken Donaldson ${ }^{1}$, Keith A. Fox ${ }^{1}$, David E. Newby ${ }^{1}$ \\ ${ }^{1}$ Centre for Cardiovascular Science, The University of Edinburgh, Edinburgh, UK \\ ${ }^{2}$ Department of Respiratory Medicine and Allergy, Umeå University, Umeå, Sweden
}

Background: Exposure to particulate air pollution may be a trigger for acute myocardial infarction. We conducted controlled exposures to dilute diesel exhaust in healthy volunteers and patients with stable coronary heart disease to determine the direct effect of air pollution on vascular function, thrombus formation and myocardial ischaemia in man.

Methods: Using a double-blind randomized crossover design, male healthy volunteers and men with prior myocardial infarction were exposed to dilute diesel exhaust $\left(300 \mu \mathrm{g} / \mathrm{m}^{3}\right)$ or filtered air during periods of rest and moderate exercise in a controlled exposure facility. In healthy volunteers, thrombus formation was measured following exposure using the Badimon ex vivo perfusion chamber. In patients, myocardial ischemia was quantified by ST-segment analysis using continuous 12-lead electrocardiography during the exposure, and vascular vasomotor and fibrinolytic function was assessed using intra-arterial agonist infusions after the exposure.

Results: Compared to filtered air, diesel exhaust inhalation increased thrombus formation under low and high shear conditions by $24.2 \%$ (95\% confidence interval [CI], $13.5 \%$ to $35.0 \%, P<0.001)$ and $19.1 \%(95 \% \mathrm{CI}, 10.5 \%$ to $27.8 \%, P<0.001)$ respectively. Exposure to diesel exhaust did not aggravate pre-existing vasomotor dysfunction in patients with coronary heart disease, but did reduce acute endothelial tissue plasminogen activator release $(P<0.05$; area under the curve decreased by $35 \%$ ). Exercise induced ST-segment depression was present in all patients but there was a greater increase in ischemic burden during exposure to diesel exhaust $(-22 \pm 4$ versus $-8 \pm 6 \mathrm{mVs}, \mathrm{P}<0.001)$.

Conclusions: Inhalation of diesel exhaust inhibits endogenous fibrinolytic capacity and increases ex vivo thrombus formation in man. Furthermore, diesel exhaust promotes exercise induced myocardial ischemia in men with stable coronary heart disease. Our findings have identified ischemic and thrombotic mechanisms for the observations that exposure to particulate air pollution is associated with adverse cardiovascular events. 